\title{
Random Indexing for Finding Similar Nodes within Large RDF Graphs
}

\author{
Danica Damljanovic ${ }^{1}$, Johann Petrak ${ }^{2}$, Mihai Lupu ${ }^{3}$, Hamish Cunningham ${ }^{1}$, \\ Mats Carlsson ${ }^{4}$, Gunnar Engstrom ${ }^{4}$, and Bo Andersson ${ }^{4}$ \\ 1 Department of Computer Science, University of Sheffield, United Kingdom \\ \{d.damljanovic,h.cunningham\}@dcs.shef .ac.uk \\ 2 Austrian Research Institute for Artificial Intelligence, Vienna, Austria \\ johann.petrak@ofai .at \\ 3 Information Retrieval Facility (IRF), Vienna, Austria \\ m.lupu@ir-facility.org \\ 4 AstraZeneca, Lund, Sweden \\ \{Mats.Carlsson, Gunnar.Engstrom, Bo.H.Andersson\}@astrazeneca.com
}

\begin{abstract}
We propose an approach for searching large RDF graphs, using advanced vector space models, and in particular, Random Indexing (RI). We first generate documents from an RDF Graph, and then index them using RI in order to generate a semantic index, which is then used to find similarities between graph nodes. We have experimented with large RDF graphs in the domain of life sciences and engaged the domain experts in two stages: firstly, to generate a set of keywords of interest to them, and secondly to judge on the quality of the output of the Random Indexing method, which generated a set of similar terms (literals and URIs) for each keyword of interest.
\end{abstract}

Keywords: random indexing, vectors space models, information retrieval, RDF graphs, ontologies.

\section{Introduction}

Recent years have seen a massive increase of highly structured data being made available in the form of RDF triple representations. Both legacy data and new data have been made available in RDF triple format and this representation has also made it worthwhile and feasible to create mappings between RDF data that originates from different legacy sources, leading to potentially very large RDF repositories. Initiatives such as Linked Open Data1 are working on creation, publication and interlinking of huge RDF graphs.

Traditionally, RDF spaces are being searched using an RDF query language such as SPARQL [15] which allows the formulation of fine-grained queries by their ability to match whole graphs and to create complex conditions on the variables to be bound in the query. This level of complexity and flexibility is

\footnotetext{
1 http://linkeddata.org/
} 
very useful in many situations, especially when the query is created automatically in the context of an application. However, for end-users who want to explore the knowledge represented in an RDF store, this level of detail is often more of a hindrance: querying the repository is not possible without a detailed knowledge of its structure and the names and semantics of all the properties and classes involved. This is especially the case for large RDF graphs which may have thousands of classes and properties, for example Linked Life Data 2 (5 billion statements), or FactForg 63 ( 2 billion statements).

In this paper we investigate whether advanced Information Retrieval (IR) methods can bring a new dimension to the task of searching huge RDF graphs. We propose a complementary approach based on word space model, more concretely Random Indexing (RI) [14, for building a semantic index for a large RDF graph. Traditionally, a semantic index captures the similarity of terms based on their contextual distribution in a large document collection, and the similarity between documents based on the similarities of the terms contained within. By creating a semantic index for an RDF graph, we are able to determine contextual similarities between graph nodes (e.g., URIs and literals) and based on these, between arbitrary subgraphs. These similarities can be used for finding a ranked list of similar URIs/literals for any given input term (a literal or a URI), which can then be used for exploring the repository or enriching SPARQL queries.

We evaluate our approach on subsets of the Linked Life Data (LLD) repository - a large integrated repository which contains 5 billion RDF statements from the biomedical domain, including UniProt 4 , PubMed5, and many more. Our evaluation is based on human judgment by clinical research scientists (from AstraZeneca pharmaceutical company) who were involved in two stages: firstly, to generate a set of keywords of interest to them, and secondly to judge on the quality of the output of the Random Indexing method, which generated a set of similar terms (literals and URIs) for each topic of interest.

\section{Related Work}

A considerable amount of work has been done in the area of using Information Retrieval methods for the task of selecting and retrieving RDF triples. However, most of these approaches do not take advantage of the latent semantics included in an RDF Graph, as their primary intention is finding the RDF files on the Web relevant to the given keyword and/or a URI. These systems are semantic search engines such as Swoogle [8] or Sindice [19]. They collect the Semantic Web resources from the Web and then index the keywords and URIs against the RDF files containing those keywords and URIs, using the inverted index scheme. These search engines use traditional weighting mechanisms such as TF-IDF.

\footnotetext{
2 WWW.linkedlifedata.com

3 WWW.factforge.net

4 www.uniprot.org/

5 wWw.ncbi.nlm.nih.gov/PubMed/

6 See the full list at: www. linkedlifedata.com/sources
} 
In 11] the authors introduce the ReConRank algorithm, which adapts the wellknown PageRank algorithm to the Semantic Web data. This method ranks the nodes in a topical subgraph that is selected based on keyword matching from the RDF files. In other words, it ranks the results of a query based on the RDF links in the results. The subgraph that the algorithm identifies includes both the subject nodes related to the query, and also the context of the subject nodes (i.e. the provenances or sources of the subjects), in order to improve the quality of ranking. In comparison to these approaches we use the neighbouring nodes as semantic context for each node in an RDF graph. The nodes and their contexts are used as virtual documents for Random Indexing.

In [16, the authors describe an approach for generating a virtual document for each URI reference in an RDF triple store (or, equivalently, each node in an RDF graph). The virtual document contains the local name and labels of the URI reference, other associated literals such as those in rdfs:comment, and the names of neighbouring nodes in the RDF graph. These virtual documents are then used for ontology matching and also for generating object recommendations for users of Falcons [2]. In comparison to our approach, their neighbouring operations involve only one-step neighbours without including properties. Our approach includes properties, and parts of the TBox, and also can operate on an arbitrarily large graph of neighbouring nodes.

While using latent semantics in the context of large RDF graphs is not widespread, some recent approaches include the TripleRank 10, algorithm, which uses 3-dimensional tensors to represent RDF properties. This approach is evaluated in the context of faceted browsing and shows promising results. Another similar work, described in [17], applies Random Indexing to Wikipedia articles and then links them to URIs in DBpedia.org.

\section{Semantic Index}

Latent Semantic Analysis (LSA) [7] is one of the pioneer methods to automatically find synonyms. The assumption behind this and other statistical semantics methods is that words which appear in the similar context (with the same set of other words) are synonyms. Synonyms tend not to co-occur with one another directly, so indirect inference is required to draw associations between words used to express the same idea [3]. This method has been shown to approximate human performance in many cognitive tasks such as the Test of English as a Foreign Language (TOEFL) synonym test, the grading of content-based essays and the categorisation of groups of concepts (see 3]). However, one problem with this method is scalability: it starts by generating a term *document matrix which grows with the number of terms and the number of documents and will thus become very large for large corpora. For finding the final LSA model, Singular Value Decomposition (SVD) and subsequent dimensionality reduction is commonly used. This technique requires the factorization of the term-document matrix which is computationally costly and does not scale well. Also, calculating the LSA model is not easily end efficiently doable in an incremental or 
out-of-memory fashion. The Random Indexing method [18] circumvents these problems by avoiding the need of matrix factorization in the first place.

RI can be seen as an approximation to LSA which is shown to be able to reach similar results (see [14] and [4]). RI can be incrementally updated and also, the term * document matrix does not have to be loaded in memory at once - loading one row at the time is enough for computing context vectors. Instead of starting with the full term-document matrix and then reducing the dimensionality, RI starts by creating almost orthogonal random vectors (index vectors) for each document. This random vector is created by setting a certain number of randomly selected dimensions to either +1 or -1 . Each term is represented by a vector (term vector) which is a combination of all index vectors of the document in which it appears. For an object consisting of multiple terms (e.g. a document or a search query with several terms), the vector of the object is the combination of the term vectors of its terms.

Random Indexing relies on the Johnson-Lindenstrauss lemma:

Lemma 1. Given $0<\epsilon<1$, a set $X$ of $m$ points in $R^{N}$, and a number $n>$ $n_{0}=O\left(\frac{\log (m)}{\epsilon^{2}}\right)$, there exists a mapping $f: R^{N} \rightarrow R^{n}$ such that $(1-\epsilon)\|u-v\| \leq$ $\|f(u)-f(v)\| \leq(1+\epsilon)\|u-v\|$, for all $u, v \in X$.

And particularly on the proof provided by Johnson and Lindenstrauss in their 1984 article [13], where they show that if one chooses at random a rank $n$ orthogonal projection, then, with positive probability, the projection restricted to $X$ will satisfy the condition in the Lemma. RI relies on the observation that, in a high dimensional space, a random set of vectors is always almost orthogonal.

In order to apply RI to an RDF graph we first generate a set of documents which represent this graph, by generating one virtual document for each URI in the graph (Section 3.1). Then, we generate a semantic index from the virtual documents (Section 3.2). This semantic index is then being searched in order to retrieve similar literals/URIs (Section 3.3).

\subsection{Generating Virtual Documents}

The task of deriving a set of documents from a huge RDF graph starts with generating a representative subgraph for each URI of interest. We shall refer to such an URI as a representative URI.

A representative subgraph represents the context of a URI i.e. the set of other URIs and literals directly or indirectly connected to that URI. For a representative URI $\mathrm{S}$, the representative subgraph of order $\mathrm{N}$ is a set of all paths of triples $\left(S, P_{1}, O_{1} ; O_{1}, P_{2}, O_{2} ; \cdots ; O_{N-1}, P_{N}, O_{N}\right)$. If $O_{N}$ is not a literal we also include all triples $O_{N}, P_{N+1}, L_{J}$ where $L_{J}$ is a literal. In other words, we apply the breadth-first search starting with the representative node, and the depth being defined by N. In addition, we include or exclude certain parts of the TBox: direct classes for instances are excluded $\left(P_{N} !=r d f:\right.$ type $)$, while other annotation properties such as $r d f s$ : label are included. In the experiments reported in this paper, the representative subgraphs are of order $1(N=1)$. 
We create virtual documents by including all paths from representative subgraphs where:

- all URIs of nodes or appearing inside literals are included unchanged;

- for literals we remove punctuation and stop words, and then lowercase the text; we also remove number literals, gene and protein sequences, complex names, and HTML tags.7.

\subsection{Generating Semantic Index}

There are several parameters which can influence the process of generating semantic index, or vectors using the RI method:

- Seed length. Number of +1 and -1 entries in a sparse random vector.

- Dimensionality. Dimension of the semantic vector space - predefined number of dimensions to use for the sparse random vectors.

- Minimum term frequency. Minimum frequency of a term to get included in the index.

Our experiments study how variations of these parameters influence the quality of the results and how sensitive the method is to that variation.

\subsection{Search}

Once the semantic index has been created, it can be used to find similarities between URIs, literals, and RDF subgraphs. We use the cosine function to calculate the similarity between the input term (literal or URI) vector and the existing vectors in the generated vector space model. We can perform the following kinds of searches:

1. finding similarities between two terms: given a keyword, find similar literals and URIs; this can be used in several ways for example for refinement of SPARQL queries (see [6]); also, it can be used as an alternative way of browsing and finding URIs or literals related to a topic of interest (expressed through a keyword or a set of keywords)

2. finding documents related to a specific term: this task would be useful for suggesting a set of representative URIs related to a given keyword.

3. finding documents related to a document: this task would be useful for suggesting a set of representative URIs related to a set of URIs.

4. finding terms related to the specific documents: this can be used for describing a representative URIs through a set of literals and URIs.

While in the context of large RDF graphs (e.g., LLD) we find all these searches useful, in the experiments we present next we focus on term-term search only.

\footnotetext{
${ }^{7}$ Although the method described in this paper is domain agnostic, carefully choosing the preprocessing strategies to suit the domain covered by an RDF graph can significantly improve results.
} 


\section{Experiments}

Our goal in using the Random Indexing method is to investigate whether it can offer an alternative way of searching large RDF spaces, by suggesting literals or URIs which are similar to the topic of interest. As the LLD dataset covers the life sciences domain, we conduct an evaluation experiment with clinical research scientists from AstraZeneca, with the aim to assess this.

\subsection{Dataset}

Linked Life Data is a dataset covering the life sciences domain, and the latest version 0.6 contains 5,052,047,661 statements in total (for a comparison, one year ago it contained 4,179,999,703 statements). Advanced IR methods based on Vector Space Model (VSM) are computationally expensive, and therefore, before we apply the Random Indexing method on the whole dataset, we evaluate it on two smaller subsets of LLD.

We have generated the two subsets as follows. For 1528 seed URIs (the URIs representing all MEDLINE articles from December 2009) we retrieve neighbouring subgraphs (of order 1) recursively until we reach certain predefined limit of statements, and we refer to these as LLD1 and LLD2. Table 1 shows the sizes of LLD1 and LLD2.

Table 1. Sizes of LLD1 and LLD2 datasets

\begin{tabular}{lll}
\hline & LLD 1 & LLD2 \\
\hline number of statements & 595798 & 4573668 \\
\hline number of virtual documents & 64644 & 473742 \\
\hline number of terms & 417753 & 1713349 \\
\hline
\end{tabular}

\subsection{Evaluation Measures}

In order to calculate the correctness of the retrieved terms, there are standard Information Retrieval measures such as precision, recall and Mean Average Precision (MAP). Precision is defined as the number of relevant documents retrieved divided by the total number of documents retrieved and is usually calculated for certain number of retrieved documents (e.g., Precision@10, Precision@20). Recall is the number of relevant documents retrieved divided by the total number of existing relevant documents (which should have been retrieved).

Mean Average Precision (MAP) is by far one of the most popular measures in IR evaluation because, for each system and set of topics, it provides a single value to measure its performance [5]. Average Precision (AP) is computed for each topic by first calculating precision for each relevant document that is retrieved and then averaging these values. Mean Average Precision is then the mean of these values for all keywords. Furthermore, by the nature of the averaging process, MAP is more sensitive to ranking than precision at a specific point, favouring systems which return more relevant documents at the top of the list 
than at the bottom, whereas precision does not make this distinction as long as the results are within the cut-off range.

As our task is to retrieve most relevant literals and URIs first, we used MAP@10. Recall is extremely difficult to measure due to the number of terms in our datasets (see Table 1). In addition, our task is to help domain experts explore large RDF graphs, which is similar to Web search in the sense that there is a vast amount of terms to be searched through, and also a significant number which is relevant for each input term. Hence, for these kinds of tasks, users care more about precision than about recall. Indeed, they care most about the top ranked results, which is exactly what is captured by MAP.

For each query keyword, two clinical research scientists evaluated the relevance of the retrieved terms. All scientists looked at all retrieved terms. Relevant were considered only those terms which both scientists evaluating a particular query marked as relevant. In order to measure the agreement between scientists on this particular task, we measured the Inter Annotator Agreement (IAA) between the two clinicians, based on the words which both of them marked as relevant/irrelevant.

IAA has been used mainly in classification tasks, where two or more annotators are given a set of instances and are asked to classify those instances into some pre-defined categories. The two commonly used IAA measures are observed agreement and Kappa $(\kappa)[12$.

Observed agreement is the portion of the instances on which the annotators agree. For our case, with the two annotators and two categories (relevant and irrelevant), it is defined as

$$
A_{o}=\frac{a+d}{a+b+c+d}
$$

where $a$ refers to the number of terms both annotators agreed as relevant, $d$ refers to the number of terms both agreed as irrelevant, $b$ refers to the number of terms annotator 1 marked as relevant, and annotator 2 as irrelevant, $c$ refer to the number of terms annotator 1 marked as irrelevant, and annotator 2 as relevant.

A certain amount of agreement is expected by chance which is not captured by the observed agreement. Kappa is defined as the observed agreements $A_{o}$ minus the agreement expected by chance $A_{e}$ and is normalized as a number between -1 and 1 .

$$
k=\frac{A_{o}-A_{e}}{1-A_{e}}
$$

$k=1$ means perfect agreement, $k=0$ means the agreement is equal to chance, $k=-1$ means 'perfect' disagreement.

There are two different methods for estimating $A_{e}$ : in Cohen's Kappa, each annotator has a personal distribution, based on his distribution of categories. In Siegel \& Castellan's Kappa, there is one distribution for all annotators, derived from the total proportion of categories assigned to all annotators (see [9] for more details and for the comparison of the two). We used Cohen's Kappa. 


\subsection{Experimental Setup}

We have performed our experiment through the following steps:

1. Extracting topics of interest represented as query terms present in both LLD1 and LLD2. In order to avoid exposing the scientists to learning SPARQL, we have formed a team of one computer scientist and one clinical research scientist. The computer scientist was executing the SPARQL query and browsing through the links and URIs, while the clinical research scientist was only looking at the abstracts which the computer scientist selected. As a result, we obtained 18 keywords which appeared in both LLD1 and LLD2 datasets. We split this set into two halves as shown in Table 2, and then performed the following two steps in two iterations: first, Group 1 is used for training the model, and Group 2 for testing it. In the second iteration, the two sets are swapped.

Table 2. Topics of interest divided into two groups for training/testing the Random Indexing method

\begin{tabular}{|c|c|}
\hline Group 1 & Group 2 \\
\hline acetylcholinesterase & Posttraumatic Stress Disorder \\
\hline synergistic effect & trial \\
\hline cholinergic signaling & bladder cancer \\
\hline PTSD & Adverse events \\
\hline antagonist & trauma \\
\hline efficacy & antioxidant \\
\hline clinical trial & magnesium \\
\hline cognitive & cystectomy \\
\hline lung & 5-HT receptors \\
\hline
\end{tabular}

2. Training the model. we generated RI models for several variations of the following RI parameters for both LLD1 and LLD2:

- vector dimension: 500, 1000, 1500, 1800, 2500

- seed length: 10, 50, 100,300, 500, 1000

- term frequency: $1,2,5,8,10$

This resulted in 290 runs (145 per datase 8 ). We then searched for the top ten similar words for each topic of interest from the training set, and presented them to clinicians who accessed the relevance. The combinations for parameters which lead to the best results (measured through MAP) were considered as the optimal setting for testing the method in the next step.

3. Testing the model. for the models generated using the best parameters retrieved in the previous step, we retrieved ten similar words for each topic of interest from the testing set and calculated MAP. The correctness of the retrieved terms was assessed by clinical research scientists to whom we gave the terms in the form of a survey (see below).

85 runs are missing from this count, corresponding to the situation where the seed size is 1000 , and the vector dimensionality is 500 , which is impossible. 
Human Assessment. The retrieved keywords for each topic of interest in both training and testing sets were assessed by humans. We merged the results from all searches into one pool (sorted alphabetically to avoid bias), and gave this list to the scientists in the form of a survey. When the similar term was a URI, we have extracted the label from LLD and showed it in brackets. This is to ensure that the scientists can concentrate on meaning of these rather than looking and searching LLD in order to find the label. An example task looked similar to this:

Is 'trauma' related to (delete URIs/words which are not related):

arteriopathy

back-projection

barotraumas

gunshot

http://linkedlifedata.com/resource/umls/id/C0003048 (Animal

Experimentation)

http://linkedlifedata.com/resource/umls/id/C0004601 (Back Injuries)

http://linkedlifedata.com/resource/umls/id/C0005604 (Birth trauma)

The most difficult task when designing this experiment was to define the meaning of relevant. Relevant, in this context, is any word related to the given keyword. This is a quite broad definition, which has, as it has been reported by clinical research scientists who were involved in this experiment, posed a number of difficulties due to many different levels of relevance. One of them stated that it would not be easy to repeat the same tasks and mark the same words as relevant if they had to repeat the same task again. that are not deleted as relevant. Therefore, only those words which have been marked as relevant twice (by two different clinicians) were eventually used when evaluating our results.

\subsection{Results}

In this section we first look into the results of training the model and finding the best parameters with two separate groups independently. Then, we look at the results of testing the RI method using these best parameters.

Training the Model. We expect to see variations of MAP, for different values of dimensionality, seed length, and minimum term frequency parameters. Our goal is to find the combination of parameters for which MAP is highest, so as to use those in the testing phase.

Figure 1 shows the distribution of MAP across all cases, and for each group used for training. It seems that the keywords from Group 1 were more challenging for the method, as MAP values are much lower on average. However, as we can see in Table 3 results for Group 1 were better with LLD2 in comparison to LLD1, while for Group 2 results were better with LLD1. The reason is a high difference in MAP for keywords: 5-HT receptors, trauma and trial. All other keywords from Group 1 performed similarly for both datasets. However, looking closely into results of Group 2 and the differences of MAP per keyword, there 

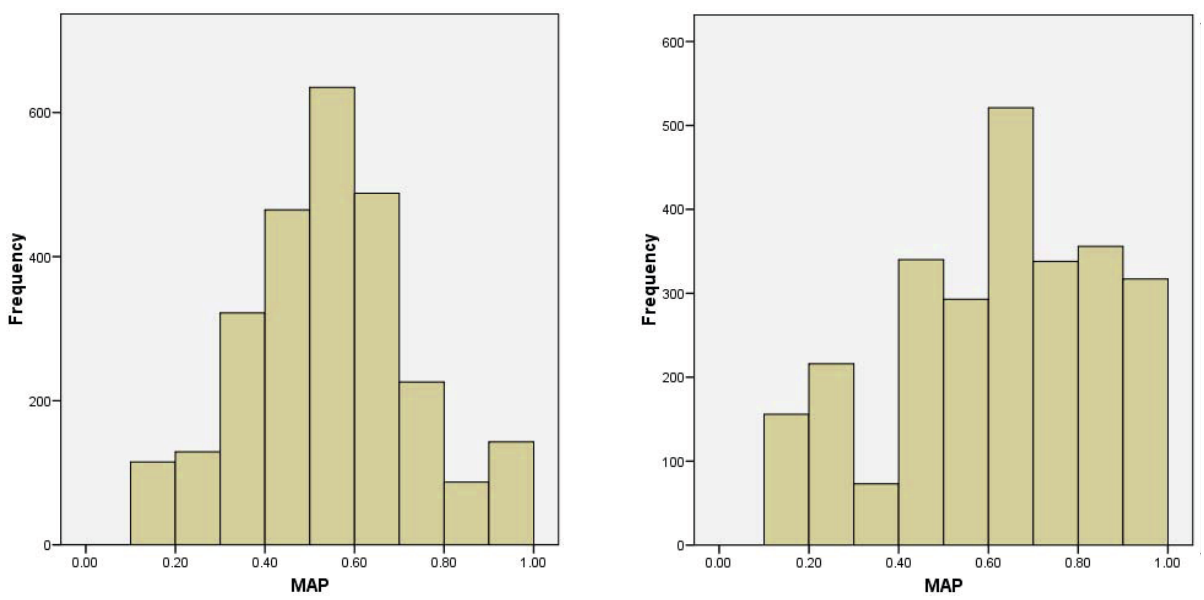

Fig. 1. The distribution of the Mean Average Precision for all combinations of parameters, for Group 1 (left) and Group 2 (right) used as training sets

is a fluctuation with one half performing better with LLD1, while the other half performing better with LLD2 (see scattergram in Figure 2).

Table 3. The dispersion values for the distribution of MAP across two datasets

\begin{tabular}{|l|c|c|c|c|}
\hline & Dataset & Number of runs & Mean & Std. Deviation \\
\hline Group 1 & 1 & 1305 & 0.50 & 0.16 \\
& 2 & 1305 & 0.59 & 0.19 \\
\hline & $1 \& 2$ & 2610 & 0.54 & 0.18 \\
\hline Group 2 & 1 & 1305 & 0.65 & 0.22 \\
& 2 & 1305 & 0.59 & 0.24 \\
\hline & $1 \& 2$ & 2610 & 0.62 & 0.23 \\
\hline
\end{tabular}

Looking closely into the effect of parameter variations, considering LLD1 and LLD2 groups independently, the results reveal that variations of the parameters did not have major influence on MAP. Detailed diagrams outlining the influence of the variation of all parameters are shown in Figures 3, 4, and 5.

Although parameter variations seem not to have a significant influence on MAP, there are certain patterns which are visible. Namely, the best minimum term frequency parameter is in the bottom range (1 for Group 2 and 2 for Group 1) for the smaller dataset, while for the larger, it seems to be in the top (10 for both groups). This might be an explanation for MAP being lower for the larger dataset: more data causes more noise which seems to be filtered nicely using the minimum frequency parameter.

With regards to dimensionality, its variation has more influence on MAP with the smaller dataset, than with the larger one. This indicates that the value span which we chose for dimensionality parameter for LLD2 needs to be expanded in 


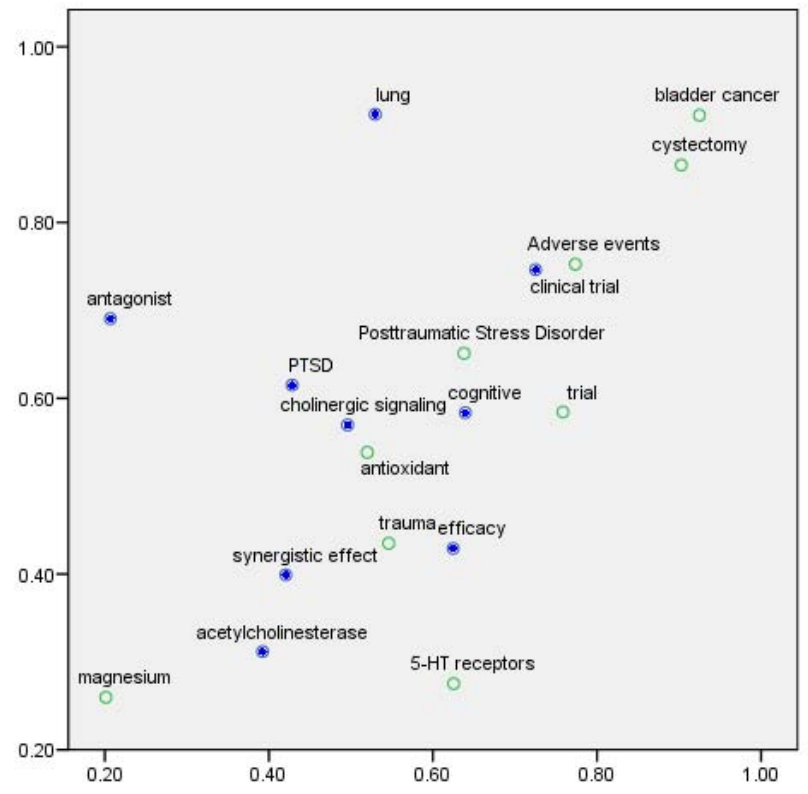

Fig. 2. Correlation of MAP for LLD1 (X axis) and LLD2 ( $\mathrm{Y}$ axis) for all keywords

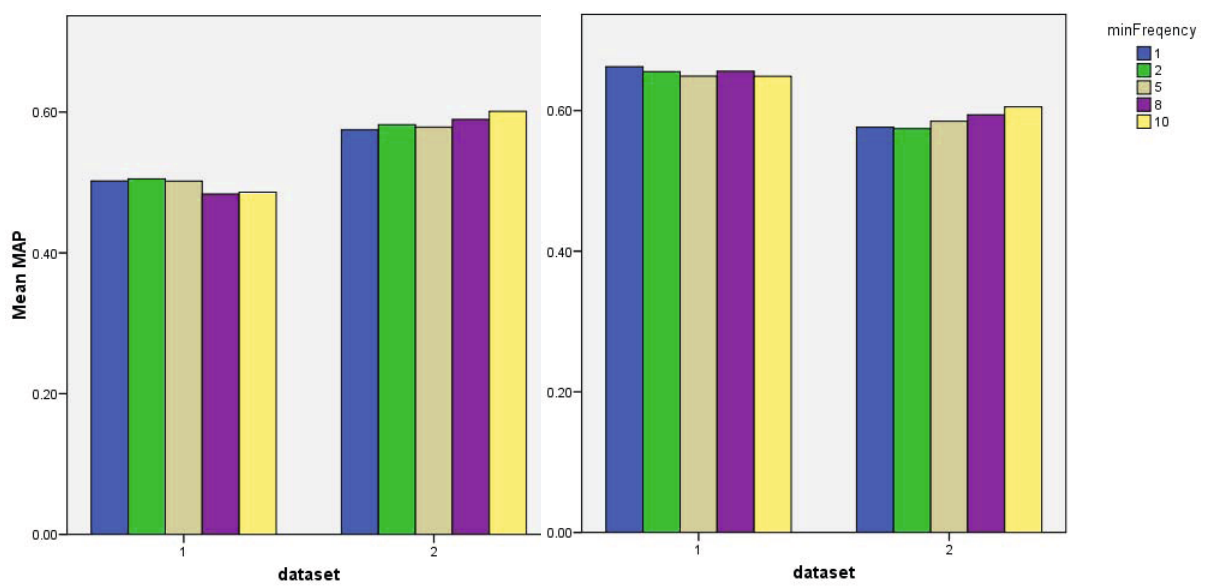

Fig. 3. The effect of the variation of minimal term frequency on $M A P$, across two datasets, for Group 1 (left) and Group 2 (right) used as training sets. The distribution of MAP across all categories of minimum term frequency is the same (independent samples Kruskal-Wallis test, $\mathrm{p}=0.44$ and $\mathrm{p}=0.444$ for LLD1 and LLD2 respectively, Group $1 ; \mathrm{p}=0.808$ and $\mathrm{p}=0.784$ for LLD1 and LLD2, Group 2). 


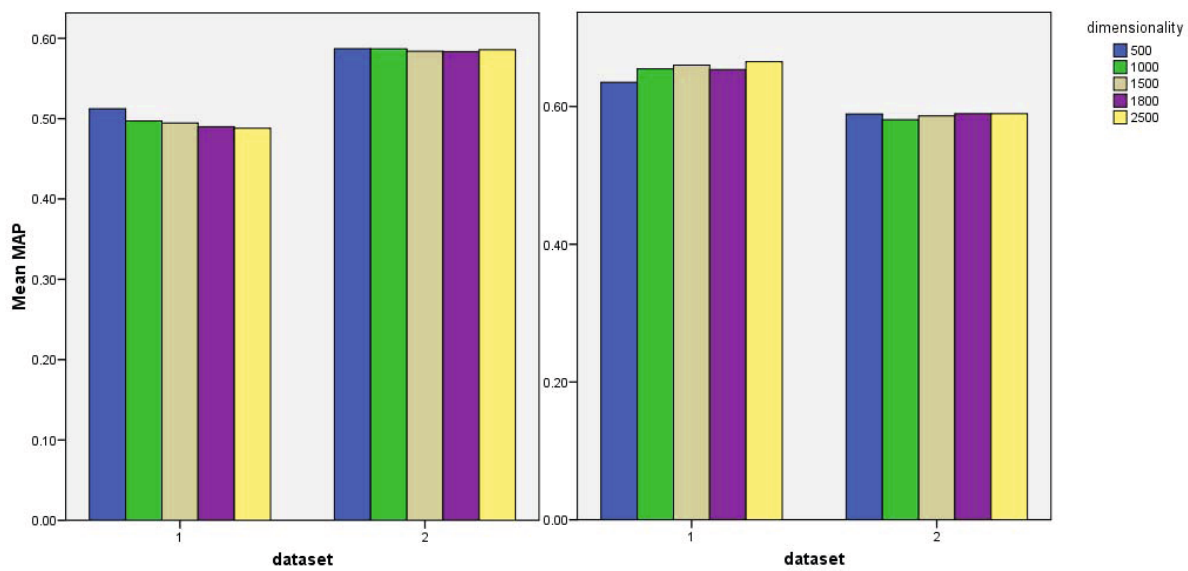

Fig. 4. The effect of the variation of dimensionality on $M A P$, across two datasets, for Group 1 (left) and Group 2 (right) used as training sets. The distribution of MAP across all categories of dimensionality is the same (independent samples Kruskal-Wallis test, $\mathrm{p}=0.676$ and $\mathrm{p}=1.0$ for LLD1 and LLD2 respectively, Group $1 ; \mathrm{p}=0.587$ and $\mathrm{p}=0.996$ for LLD1 and LLD2, Group 2).
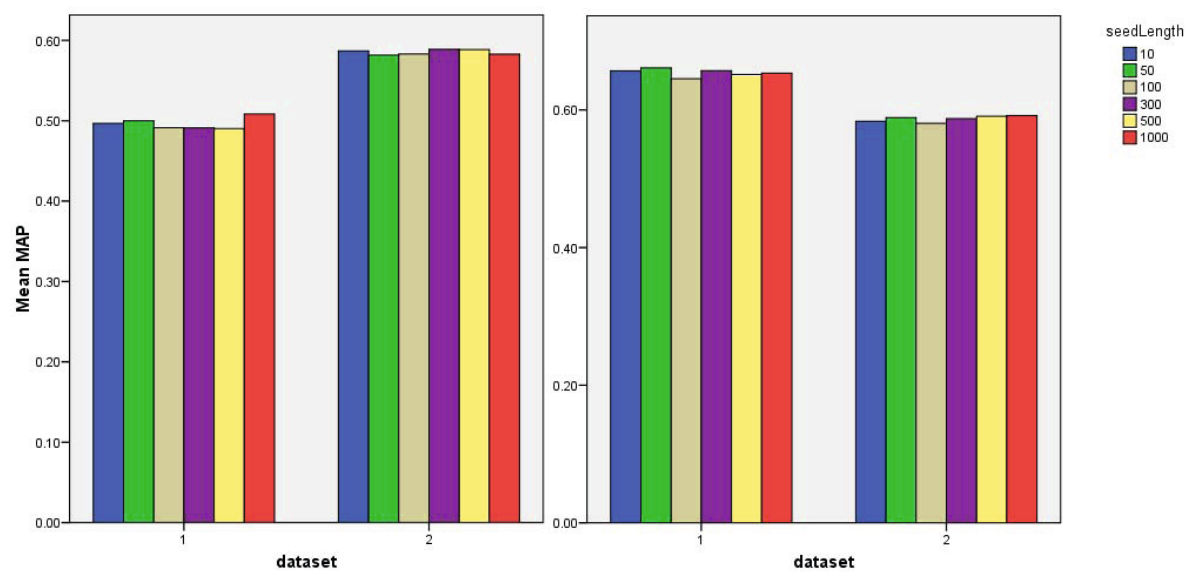

Fig. 5. The effect of the variation of seed length on $M A P$, across two datasets, for Group 1 (left) and Group 2 (right) used as training sets. The distribution of MAP across all categories of minimum term frequency is the same (independent samples Kruskal-Wallis test, $\mathrm{p}=0.931$ and 0.997 for LLD1 and LLD2 respectively, Group 1; $\mathrm{p}=0.961$ and 0.998 for LLD1 and LLD2, Group 2).

order to have any effect on results. However, MAP values are within reasonable range.

The variation of seed length parameter value seems not to cause any significant changes to MAP across both datasets, and hence, we consider the lowest value of this parameter as the optimal one, due to the fact that the computational 
Table 4. Optimal parameters chosen for Group 1 and Group 2 used as training sets

\begin{tabular}{|l|ll|ll|}
\hline & Group 1 & Group 2 \\
\hline Dataset & LLD1 & LLD2 & LLD1 & LLD2 \\
\hline Min frequency & 2 & 10 & 1 & 10 \\
\hline Seed length & 10 & 10 & 10 & 10 \\
\hline Dimensionality & 500 & 500 & 1500 & 500 \\
\hline MAP & 0.55 & 0.61 & 0.65 & 0.61 \\
\hline
\end{tabular}

resources required to build and search the semantic space are proportional to the value of seed length. Table 4 outlines optimal parameters: those that we chose to use in the testing phase.

Finally, the size of the dataset had a significant influence on MAP (MannWhitney U Test, $p<0.0001$ ) for both Group 1 and 2 meaning that the larger set (LLD2) resulted in producing the higher value of MAP for Group 1, while for Group 2 the results were better with the smaller dataset (LLD1).

Testing the Model. In what follows we explore whether the model built using the optimal parameters just presented can be used to effectively test the model. In our context, testing the model means evaluating the set of related terms (literals and URIs) returned by our method for the set of testing keywords given as input.

We ran the search method using Group 2 as a testing set against the RI model trained with Group 1, and then Group 1 as a testing set against the RI model trained with Group 2. Results are shown in Table 5. The RI method results in as good or better MAP for Group 2 in comparison to MAP for the best trained model (Group 1 column in Table 4), while for Group 1 the resulting MAP for LLD2 is as good as that of the best trained model (Group 2 column in Table 4), while for LLD1 it is lower for 0.15. This is due to the distribution of keywords in Group 1, due to which MAP for the RI model with optimal parameters is only 0.05 higher $(0.55)$.

In the testing phase, MAP across both groups reached 0.565 and 0.61 for LLD1, and LLD2 respectively.

Table 5. Testing the Random Indexing method using Group 2 and Group 1 as testing sets

\begin{tabular}{|l|ll|ll|}
\hline & Group 2 & Group 1 \\
\hline Dataset & LLD1 & LLD2 & LLD1 & LLD2 \\
\hline Min frequency & 2 & 10 & 1 & 10 \\
\hline Seed length & 10 & 10 & 10 & 10 \\
\hline Dimensionality & 500 & 500 & 1500 & 500 \\
\hline MAP & 0.63 & 0.61 & 0.5 & 0.61 \\
\hline
\end{tabular}

Also important to observe is the fact that when the data corpus increases (e.g. LLD2 vs. LLD1) the method becomes very stable, and observed MAP values in the training process are reproduced in the subsequent test phase. Arguably this 
is due to the small difference in MAP across parameters, but it still shows that $\mathrm{RI}$ is a stable method even in this unusual use-case we are dealing with.

Human Assessment. In order to assess the overall difficulty of the task which we solve using the RI method, we calculated Inter-annotator agreement, and indeed Observed agreement and Cohen's Kappa agreement (see Section 4.2). The observed agreement across all keywords was 0.81, and the Cohen's Kappa was 0.61 which indicates that the given task of selecting relevant keywords for a topic of interest was indeed difficult for domain experts.

The code and datasets from the described experiment, including generated virtual documents and semantic spaces, can be downloaded from the LarKC Wik!.

Performance. The parameter values affect not only the quality of results but also the required resources and the indexing time. Increasing the value of dimensionality and seed length almost exponentially increases the time to generate the semantic space (from 0.67 minutes for 500 dimensions to 3 minutes for 2500 , LLD1; from 3.78 minutes for 500 dimensions to 11.5 minutes for 2500, LLD2). The higher the value for seed length and dimensionality, the higher the requirements for the computational resources and RAM in particular 10 . Application of RI to the whole LLD dataset poses the scalability issues related to the size of our corpus. While indexing is a one-off operation (that takes $\tilde{1} 6$ hours on MDC computer with 256G RAM), the search for 'lung' after the space is generated takes 14 minutes. Therefore, in our related work reported elsewhere [1] we looked at the parallelisation of the RI search algorithm in order to make exploring large RDF graphs using the contextual similarities of the comprising nodes applicable in real time applications.

\section{Conclusion and Future Work}

We described the application of the Random Indexing method for the task of searching large and unknown RDF graphs. We tested our method in the domain of life sciences, by training it using the variation of parameters, and then involving domain experts to judge on the relevance of retrieved terms. None of the parameters had a significant influence on MAP, apart from the size of the dataset. However, the value of MAP reaching 0.59 on average indicates that the generation of virtual documents as described in this paper and generating the semantic index using the RI method has promising results, especially considering that the human agreement on the same task revealed its difficulty for domain experts. The reason for the stability of the RI method might have been the span of the parameters which we used, and hence in our future work we will expand the variation span and also repeat the runs across the same parameter variations in order to increase the significance of results.

\footnotetext{
9 http://wiki.larkc.eu/LarkcProject/statisticalSemantics

10 The experiments are conducted on the MDC super-computer: 2 IBM x3950M2, 32 Cores, 256 Gbytes of main memory.
} 
Acknowledgments. We would like to thank creators of the SemanticVectors 11 library which is used in the experiments reported in this paper. This research has been supported by the EU-funded LarKC12 (FP7-215535) project.

\section{References}

1. Assel, M., Cheptsov, A., Czink, B., Damljanovic, D., Quesada, J.: MPI Realization of High Performance Search for Querying Large RDF Graphs using Statistical Semantics. In: García-Castro, R., et al. (eds.) ESWC 2011 Workshops. LNCS, vol. 7117, pp. 156-171. Springer, Heidelberg (2011)

2. Cheng, G., Ge, W., Qu, Y.: Falcons: Searching and Browsing Entities on the Semantic Web. In: Proceedings of WWW 2008, pp. 1101-1102 (2008)

3. Cohen, T., Schvaneveldt, R., Widdows, D.: Reflective random indexing and indirect inference: A scalable method for discovery of implicit connections. Journal of Biomedical Informatics (2009)

4. Cohen, T.: Exploring medline space with random indexing and pathfinder networks. In: AMIA.. Annual Symposium proceedings / AMIA Symposium, pp. 126130 (2008)

5. Croft, B., Metzler, D., Strohman, T.: Search Engines: Information Retrieval in Practice, 1st edn. Addison Wesley (2009)

6. Damljanovic, D., Petrak, J., Cunningham, H.: Random Indexing for Searching Large RDF Graphs. In: Aroyo, L., Antoniou, G., Hyvönen, E., ten Teije, A., Stuckenschmidt, H., Cabral, L., Tudorache, T. (eds.) ESWC 2010. LNCS, vol. 6088, pp. 1-32. Springer, Heidelberg (2010)

7. Deerwester, S., Dumais, S., Furnas, G., Landauer, T., Harshman, R.: Indexing by latent semantic analysis. Journal of the American Society for Information Science 41, 391-407 (1990)

8. Ding, L., Finin, T., Joshi, A., Pan, R., Cost, R.S., Peng, Y., Reddivari, P., Doshi, V., Sachs, J.: Swoogle: a search and metadata engine for the semantic web. In: Proceedings of the 13th ACM International Conference on Information and Knowledge Management, pp. 652-659. ACM, New York (2004)

9. Eugenio, B.D., Glass, M.: The kappa statistic: a second look. Computational Linguistics 1(30) (2004) (squib)

10. Franz, T., Schultz, A., Sizov, S., Staab, S.: TripleRank: Ranking semantic web data by tensor decomposition. In: Bernstein, A., Karger, D.R., Heath, T., Feigenbaum, L., Maynard, D., Motta, E., Thirunarayan, K. (eds.) ISWC 2009. LNCS, vol. 5823, pp. 213-228. Springer, Heidelberg (2009), http://dx.doi.org/10.1007/978-3-642-04930-9_14

11. Hogan, A., Harth, A., Decker, S.: Reconrank: A scalable ranking method for semantic web data with context. In: Second International Workshop on Scalable Semantic Web Knowledge Base Systems (SSWS 2006), Athens, GA, USA (2006)

12. Hripcsak, G., Heitjan, D.: Measuring agreement in medical informatics reliability studies. Journal of Biomedical Informatics 35, 99-110 (2002)

13. Johnson, W.B., Lindenstrauss, J.: Extensions to lipschiz mapping into hilbert space. Contemporary Mathematics 26 (1984)

$1 1 \longdiv { \text { WWW.code.google.com/p/semanticvectors/ } }$

12 WWW.larkc.eu 
14. Karlgren, J., Sahlgren, M.: From words to understanding. In: Uesaka, Y., Kanerva, P., Asoh, H. (eds.) Foundations of Real-World Intelligence, pp. 294-308. CSLI Publications, Stanford (2001)

15. Prud'hommeaux, E., Seaborne, A.: SPARQL Query Language for RDF. W3C Recommendation. W3C (January 15, 2008)

16. Qu, Y., Hu, W., Cheng, G.: Constructing virtual documents for ontology matching. In: Proceedings of WWW 2006, pp. 23-31 (2006)

17. Quesada, J., Brandao-Vidal, R., Schooler, L.: Random indexing spaces for bridging the human and data webs. In: d'Amato, C., Fanizzi, N., Grobelnik, M., Lawrynowicz, A., Svatek, V. (eds.) IRMLeS 2010: The 2nd ESWC Workshop on Inductive Reasoning and Machine Learning for the Semantic Web (2010)

18. Sahlgren, M.: An introduction to random indexing. In: Methods and Applications of Semantic Indexing Workshop at the 7th International Conference on Terminology and Knowledge Engineering, TKE 2005. Citeseer (2005)

19. Tummarello, G., Delbru, R., Oren, E.: Sindice.com: Weaving the Open Linked Data. In: Aberer, K., Choi, K.-S., Noy, N., Allemang, D., Lee, K.-I., Nixon, L.J.B., Golbeck, J., Mika, P., Maynard, D., Mizoguchi, R., Schreiber, G., Cudré-Mauroux, P. (eds.) ASWC 2007 and ISWC 2007. LNCS, vol. 4825, pp. 552-565. Springer, Heidelberg (2007) 